Proyecciones Journal of Mathematics

Vol. 35, $\mathrm{N}^{o}$ 1, pp. 67-83, March 2016.

Universidad Católica del Norte

Antofagasta - Chile

\title{
The forcing open monophonic number of a graph
}

\author{
A. P. Santhakumaran \\ Hindustan University, India \\ and \\ M. Mahendran \\ Hindustan University, India \\ Received : April 2015. Accepted : March 2016
}

\begin{abstract}
For a connected graph $G$ of order $n \geq 2$, and for any minimum open monophonic set $S$ of $G$, a subset $T$ of $S$ is called a forcing subset for $S$ if $S$ is the unique minimum open monophonic set containing $T$. A forcing subset for $S$ of minimum cardinality is a minimum forcing subset of $S$. The forcing open monophonic number of $S$, denoted by $f_{\text {om }}(S)$, is the cardinality of a minimum forcing subset of $S$. The forcing open monophonic number of $G$, denoted by $f_{\text {om }}(G)$, is $f_{\text {om }}(G)=\min \left(f_{\text {om }}(S)\right)$, where the minimum is taken over all minimum open monophonic sets in $G$. The forcing open monophonic numbers of certain standard graphs are determined. It is proved that for every pair $a, b$ of integers with $0 \leq a \leq b-4$ and $b \geq 5$, there exists a connected graph $G$ such that $f_{\text {om }}(G)=a$ and om $(G)=b$. It is analyzed how the addition of a pendant edge to certain standard graphs affects the forcing open monophonic number.
\end{abstract}

2010 Mathematics Subject Classification : 05C12, 05C70.

Key Words : Monophonic number, open monophonic number, forcing monophonic number, forcing open monophonic number. 


\section{Introduction}

By a graph $G=(V(G), E(G))$ we mean a finite, undirected connected graph without loops or multiple edges. The order and size of $G$ are denoted by $n$ and $m$, respectively. For basic graph theoretic terminology we refer to Harary [5]. The distance, geodesic and geodetic number of a graph were introduced and studied in [1,3]. A set $S$ of vertices of a connected graph $G$ is an open geodetic set of $G$ if for each vertex $v$ in $G$, either $v$ is an extreme vertex of $G$ and $v \in S$, or $v$ is an internal vertex of a $x$ - $y$ geodesic for some $x, y \in S$. An open geodetic set of minimum cardinality is a minimum open geodetic set and this cardinality is the open geodetic number og $(G)$. The open geodetic number of a graph was introduced and studied in $[2,6,8]$. A chord of a path $u_{1}, u_{2}, \ldots, u_{n}$ in $G$ is an edge $u_{i} u_{j}$ with $j \geq i+2$. For two vertices $u$ and $v$ in a connected graph $G$, a $u-v$ path is called a monophonic path if it contains no chords. A monophonic set of $G$ is a set $S \subseteq V(G)$ such that every vertex of $G$ is contained in a monophonic path joining some pair of vertices in $S$. The monophonic number $m(G)$ of $G$ is the cardinality of a minimum monophonic set. A subset $T$ of $S$ is called a forcing subset for $S$ if $S$ is the unique minimum monophonic set containing $T$. A forcing subset for $S$ of minimum cardinality is a minimum forcing subset of $S$. The forcing monophonic number of $\mathrm{S}$, denoted by $f_{m}(S)$, is the cardinality of a minimum forcing subset of $S$. The forcing monophonic number of G, denoted by $f_{m}(G)$, is $f_{m}(G)=\min \left(f_{m}(S)\right)$, where the minimum is taken over all minimum monophonic sets in $G$.

A set $S$ of vertices in a connected graph $G$ is an open monophonic set if for each vertex $v$ in $G$, either $v$ is an extreme vertex of $G$ and $v \in S$, or $v$ is an internal vertex of a $x-y$ monophonic path for some $x, y \in S$. An open monophonic set of minimum cardinality is a minimum open monophonic set and this cardinality is the open monophonic number om $(G)$ of $G$. The open monophonic number of a graph was introduced and further studied in $[9,10,11,12]$. The following is the motivation for the introduction of open monophonic concepts and the corresponding forcing concepts. In a communication network, the main problem of protection from intercepting and overhearing is an inevitable one. Hence there is a need for enhancing security in a communication network design. Since graphs lend themselves naturally as models for these real life situations, the security based communication network problems can be studied and settled using the open monophonic and the corresponding forcing concepts. Further, these concepts have interesting applications in Channel Assignment Prob- 
lem in Radio Technologies [4].

The following theorems are used in the sequel.

Theorem 1.1. [10] Every extreme vertex of a connected graph $G$ belongs to each open monophonic set of $G$. In particular, if the set $S$ of all extreme vertices of $G$ is an open monophonic set of $G$, then $S$ is the unique minimum open monophonic set of $G$.

Theorem 1.2. [12] For a connected graph $G$ of order at least $2, o m(G)=2$ if only if there exist two extreme vertices $u$ and $v$ such that every vertex lies on a monophonic path joining $u$ and $v$.

\section{Forcing open monophonic number of a graph}

Definition 2.1. Let $G$ be a connected graph with at least two vertices and $S$ a minimum open monophonic set of $G$. A subset $T$ of $S$ is called a forcing subset for $S$ if $S$ is the unique minimum open monophonic set containing $T$. A forcing subset for $S$ of minimum cardinality is a minimum forcing subset of $S$. The forcing open monophonic number of $S$, denoted by $f_{\text {om }}(S)$, is the cardinality of a minimum forcing subset of $S$. The forcing open monophonic number of $G$, denoted by $f_{\text {om }}(G)$, is $f_{\text {om }}(G)=\min \left(f_{\text {om }}(S)\right)$, where the minimum is taken over all minimum open monophonic sets in $G$.

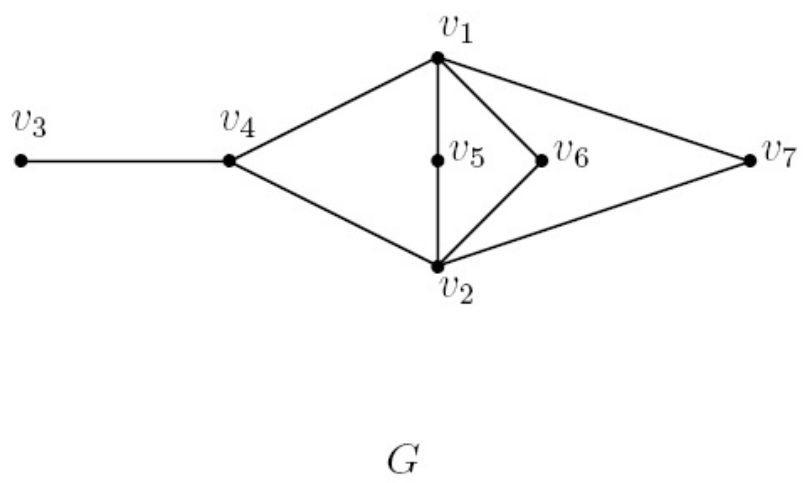

Figure 2.1.

Example 2.2. For the graph $G$ in Figure 2.1, it is clear that the sets $S_{1}=$ $\left\{v_{1}, v_{2}, v_{3}, v_{6}\right\}$ and $S_{2}=\left\{v_{1}, v_{2}, v_{3}, v_{7}\right\}$ are the minimum open monophonic 
sets of $G$. Now, $T=\left\{v_{6}\right\}$ is a forcing subset for $S_{1}$ and so $f_{\text {om }}\left(S_{1}\right)=1$. Similarly $T^{\prime}=\left\{v_{7}\right\}$ is a forcing subset for $S_{2}$ so that $f_{\text {om }}\left(S_{2}\right)=1$. Hence $f_{\text {om }}(G)=1$.

The next theorem follows immediately from the definition of an open monophonic number and the forcing open monophonic number of a connected graph $G$.

Theorem 2.3. For every connected graph $G, 0 \leq f_{o m}(G) \leq o m(G) \leq n$.

Remark 2.4. For the complete graph $G=K_{n}(n \geq 2)$, the set of all vertices of $K_{n}$ is the unique minimum open monophonic set so that $o m(G)=n$ and $f_{\text {om }}(G)=0$. Also, all the inequalities in Theorem 2.3 can be strict. For the graph $G$ given in Figure 2.1, om $(G)=4, f_{o m}(G)=1$ and $n=6$.

Now, we proceed by proving a main result regarding forcing monophonic number of a graph.

Theorem 2.5. Let $G$ be a connected graph. Then

(i) $f_{m}(G)$ (or $\left.f_{o m}(G)\right)=0$ if and only if $G$ has a unique minimum (open) monophonic set.

(ii) $f_{m}(G)$ (or $\left.f_{\text {om }}(G)\right)=1$ if and only if $G$ has at least two minimum (open) monophonic sets, one of which is the unique minimum monophonic set contains one of its elements.

(iii) $f_{m}(G)$ (or $\left.f_{o m}(G)\right)=m(G)$ (or om $(G)$ ) if and only if no minimum (open) monophonic set of $G$ is the unique minimum (open) monophonic set containing any of its proper subsets.

Proof. We prove the theorem for forcing monophonic number. The proof for open forcing monophonic number is similar.

(i) Let $f_{m}(G)=0$. Then by definition, $f_{m}(S)=0$ for some minimum monophonic set $S$ of $G$ so that the empty set $\phi$ is the minimum forcing subset for $S$. Hence it follows that $S$ is the unique minimum monophonic set of $G$. The converse is clear.

(ii) Let $f_{m}(G)=1$. Then by (i), $G$ has at least two minimum monophonic sets. Also, since $f_{m}(G)=1$, there is a singleton subset $T$ of a minimum monophonic set $S$ of $G$ such that $T$ is not a subset of any other 
minimum monophonic set of $G$. Thus $S$ is the unique minimum monophonic set containing one of its elements. The converse is clear.

(iii) Let $f_{m}(G)=m(G)$. Then $f_{m}(S)=m(G)$ for every monophonic set $S$ in $G$. Also, $m(G) \geq 2$ and hence $f_{m}(G) \geq 2$. Then by (i) $G$ has at least two minimum monophonic sets, and so the empty set $\phi$ is not a minimum forcing subset for any minimum monophonic set of $G$. Since $f_{m}(G)=m(G)$, no proper subset of $S$ is a forcing subset of $S$. Thus no minimum monophonic set of $G$ is the unique minimum monophonic set containing any of its proper subsets. Conversely, assume that no minimum monophonic set of $G$ is the unique minimum monophonic set containing any of its proper subsets. This shows that $G$ contains more than one minimum monophonic set, and no subset of any minimum monophonic set $S$ other than $S$ is a forcing subset for $S$. Hence it follows that $f_{m}(G)=m(G)$.

Definition 2.6. A vertex $v$ of a connected graph $G$ is said to be an open monophonic vertex of $G$ if $v$ belongs to every minimum open monophonic set of $G$.

We illustrate this by an example. For the graph $G$ given in Figure 2.2 , it is easily verified $S_{1}=\left\{v_{1}, v_{2}, v_{3}, v_{6}\right\}, S_{2}=\left\{v_{1}, v_{2}, v_{3}, v_{5}\right\}, S_{3}=$ $\left\{v_{1}, v_{2}, v_{3}, v_{4}\right\}, S_{4}=\left\{v_{1}, v_{2}, v_{5}, v_{6}\right\}, S_{5}=\left\{v_{1}, v_{2}, v_{4}, v_{6}\right\}, S_{6}=\left\{v_{1}, v_{2}, v_{4}, v_{5}\right\}$ are the minimum open monophonic sets. Hence the vertices $v_{1}$ and $v_{2}$ are the open monophonic vertices. It is to be noted that the set $T=\left\{v_{3}, v_{6}\right\}$ is a forcing subset for $S_{1}$ and so $f_{o m}\left(S_{1}\right)=2$. Hence $f_{o m}(G)=2$. Also, every endvertex in a graph is an open monophonic vertex.

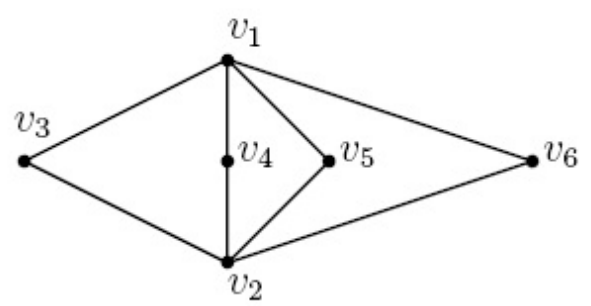

$G$

Figure 2.2. 
Theorem 2.7. Let $G$ be a connected graph and let $F$ be the set of relative complements of the minimum forcing subsets in their respective minimum open monophonic sets in $G$. Then $\bigcap_{F \in F} F$ is the set of open monophonic vertices of $G$.

Proof. Let $W$ be the set of all open monophonic vertices of $G$. If $v \in W$, then $v$ belongs to every minimum open monophonic set $S$ of $G$. Let $T \subseteq S$ be any minimum forcing subset for any minimum open monophonic set $S$ of $G$. We claim that $v \notin T$. If $v \in T$, then $T^{\prime}=T-\{v\}$ is a proper subset of $T$ such that $S$ is the unique minimum open monophonic set containing $T^{\prime}$ so that $T^{\prime}$ is a forcing subset for $S$ with $\left|T^{\prime}\right|<|T|$, which is a contradiction because $T$ is a minimum forcing subset for $S$. Thus $v \notin T$ and so $v \in F$, where $F$ is the relative complement of $T$ in $S$. Thus $v \in \bigcap_{F \in F} F$ so that $W \subseteq \bigcap_{F \in F} F$. Now, if $v \in \bigcap_{F \in F} F$, then $v$ belongs to the relative complement of $T$ in $S$ for every $T$ and every $S$ such that $T \subseteq S$, where $T$ is a minimum forcing subset for $S$. Since $F$ is the relative complement of $T$ in $S$, we have $F \subseteq S$ and thus $v \in S$ for every $S$. Hence $v$ is an open monophonic vertex of $G$ so that $v \in W$. It follows that $\bigcap_{F \in F} F \subseteq W$, and so $W=\bigcap_{F \in F} F$.

Theorem 2.8. Let $G$ be a connected graph and $W$ be the set of all open monophonic vertices of $G$. Then $f_{\text {om }}(G) \leq$ om $(G)-|W|$.

Proof. Let $S$ be any minimum open monophonic set of $G$. Then $\operatorname{om}(G)=|S|$. Since $W$ is the set of all open monophonic vertices of $G$, it follows that $W \subseteq S$ and $S$ is the unique minimum open monophonic set containing $S-W$. Hence $f_{\text {om }}(G) \leq|S-W|=|S|-|W|=o m(G)-|W|$.

The following result is an easy consequence of Theorems 1.1 and 2.8.

Corollary 2.9. If $G$ is a connected graph with $k$ extreme vertices, then $f_{\text {om }}(G) \leq o m(G)-k$.

We observe that the bound in Theorem 2.8 is sharp. For the graph $G$ given in Figure 2.2, om $(G)=4, f_{o m}(G)=2$ and $W=\left\{v_{1}, v_{3}\right\}$ is the set of open monophonic vertices of $G$. Hence $f_{o m}(G)=o m(G)-|W|$. Also, the inequality in Theorem 2.11 can be strict. For the graph $G$ given in Figure 2.3 , it is clear that $S_{1}=\left\{v_{1}, v_{3}, v_{5}\right\}$ and $S_{2}=\left\{v_{4}, v_{6}, v_{7}\right\}$ are the only two 
minimum open monophonic sets of $G$ so that $\operatorname{om}(G)=3, f_{\text {om }}(G)=1$ and $W=\phi$. Hence $f_{\text {om }}(G)<o m(G)-|W|$.

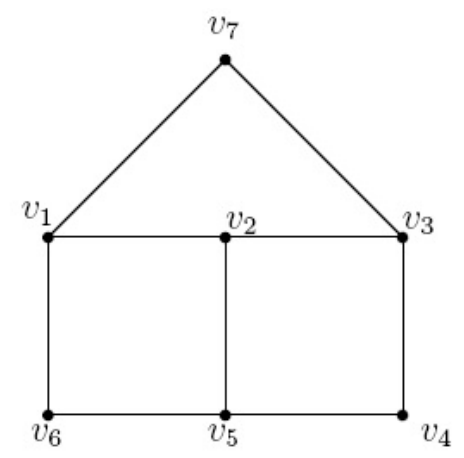

G

Figure 2.3.

Theorem 2.10. For any cycle $G=C_{n}(n \geq 4)$,

$$
f_{o m}(G)= \begin{cases}3 & \text { if } n \geq 7 \\ 1 & \text { if } n=6 \\ 4 & \text { if } n=5 \\ 0 & \text { if } n=4\end{cases}
$$

Proof. Let $G=C_{n}(n \geq 4)$ be the cycle $C_{n}: v_{1}, v_{2}, v_{3}, \ldots, v_{n}, v_{1}$.

For $n=4, S=\left\{v_{1}, v_{2}, v_{3}, v_{4}\right\}$ is the unique open monophonic set of $G$ so that by Theorem $2.5(\mathrm{i}), f_{\text {om }}(G)=0$.

For $n=5$, the sets $S_{1}=\left\{v_{1}, v_{2}, v_{3}, v_{4}\right\}, S_{2}=\left\{v_{1}, v_{2}, v_{3}, v_{5}\right\}, S_{3}=$ $\left\{v_{1}, v_{2}, v_{4}, v_{5}\right\}, S_{4}=\left\{v_{2}, v_{3}, v_{4}, v_{5}\right\}, S_{5}=\left\{v_{1}, v_{3}, v_{4}, v_{5}\right\}$ are the minimum open monophonic sets of $G$. It is clear that no proper subset of any of the sets $S_{i}(i=1,2,3,4,5)$ is a forcing subset so that $f_{\text {om }}(G)=4$.

For $n=6$, the sets $S_{1}=\left\{v_{1}, v_{3}, v_{5}\right\}$ and $S_{2}=\left\{v_{2}, v_{4}, v_{6}\right\}$ are the only two minimum open monophonic sets of $G$. Since $\left\{v_{1}\right\}$ is a forcing subset for $S_{1}$, it follows that $f_{\text {om }}(G)=1$.

Let $G=C_{n}(n \geq 7)$. Then any set containing three independent vertices of $G$ is a minimum open monophonic set so that $\operatorname{om}(G)=3$. Since $n \geq 7$, it is clear that no 2-element subset of any minimum open monophonic set $S$ is a forcing subset for $S$. Hence $f_{o m}(G)=3$. 
Theorem 2.11. For any wheel $W_{n}=K_{1}+C_{n-1}(n \geq 4)$,

$$
f_{\text {om }}\left(W_{n}\right)= \begin{cases}3 & \text { if } n \geq 8 \\ 1 & \text { if } n=7 \\ 4 & \text { if } n=6 \\ 0 & \text { if } n=4,5\end{cases}
$$

Proof. Let $C_{n-1}: v_{1}, v_{2}, \ldots, v_{n-1} v_{1}$. Let $K_{1}=\{x\}$. Then $W_{n}=$ $C_{n-1}+K_{1}$

For $n=4, W_{4}$ is the complete graph $K_{4}$ so that $f_{o m}(G)=0$.

For $n=5$, the set $S=\left\{v_{1}, v_{2}, v_{3}, v_{4}\right\}$ is the unique open monophonic set of $G$, then by Theorem $2.5(\mathrm{i}), f_{\text {om }}(G)=0$.

For $n=6$, it is clear that no 3 -element subset of vertices of $W_{6}$ is an open monophonic set of $W_{6}$. The sets $S_{1}=\left\{v_{1}, v_{2}, v_{3}, v_{4}\right\}, S_{2}=\left\{v_{1}, v_{2}, v_{3}, v_{5}\right\}$, $S_{3}=\left\{v_{1}, v_{2}, v_{4}, v_{5}\right\}, S_{4}=\left\{v_{2}, v_{3}, v_{4}, v_{5}\right\}$ and $S_{5}=\left\{v_{1}, v_{3}, v_{4}, v_{5}\right\}$ are the minimum open monophonic sets of $G$. It is easily verified that no subset of $S_{i}(1 \leq i \leq 5)$ containing at most 3 elements is a forcing subset for $S_{i}$. Hence it follows that $f_{o m}\left(W_{6}\right)=4$.

For $n=7$, the sets $S_{1}=\left\{v_{1}, v_{3}, v_{5}\right\}$ and $S_{2}=\left\{v_{2}, v_{4}, v_{6}\right\}$ are the only two minimum open monophonic sets of $W_{7}$. Since $\left\{v_{1}\right\}$ is a forcing subset for $S_{1}$, it follows that $f_{o m}\left(W_{7}\right)=1$.

Let $W_{n}=K_{1}+C_{n-1}(n \geq 8)$. Then any set containing three independent vertices of $W_{n}$ is a minimum open monophonic set so that $\operatorname{om}\left(W_{n}\right)=3$. Since $n \geq 8$, it is clear that no 2-element subset of any minimum open monophonic set $S$ is a forcing subset for $S$. Hence $f_{\text {om }}(G)=3$.

Theorem 2.12. For the complete bipartite graph $G=K_{r, s}(2 \leq r \leq s)$,

$$
f_{\text {om }}(G)= \begin{cases}0 & \text { if } r=s=2 \\ 2 & \text { if } 2=r<s \\ 4 & \text { if } 3 \leq r \leq s\end{cases}
$$

Proof. Case 1. $r=s=2$. Then $G$ is the cycle $C_{4}$ and by Theorem $2.10, f_{\text {om }}(G)=0$.

Case 2. $2=r<s$. Let $U$ and $W$ be the partite sets of $K_{r, s}$ with $|U|=r$ and $|S|=s$. It is clear that the minimum open monophonic sets $S$ of $G$ are obtained by choosing the two elements of $U$ and any two elements from $W$. It is easily verified that no single vertex subset of any of these sets is a forcing subset for $S$. Also, it is clear that any 2-element subset of $W$ is a forcing subset for one of these minimum open monophonic sets $S$ of $G$. Hence $f_{\text {om }}(G)=2$. 
Case 3. $3 \leq r \leq s$. Then any minimum open monophonic set $S$ is got by taking any two elements from $U$ and any two elements from $W$. It is easily verified that no 3 -element subset of any minimum open monophonic sets $S$ of $G$ is a forcing set for $S$. Hence it follows that $f_{\text {om }}(G)=4$.

It was already seen that $f_{o m}(G)=0$ if $G$ is a complete graph or a tree or the cycle $C_{4}$ or the wheels $W_{4}$ and $W_{5}$. Hence we leave the following problem as an open question.

Problem 2.13. Characterize graphs $G$ for which (i) $f_{\text {om }}(G)=0$ (ii) $f_{\text {om }}(G)=$ om $(G)$.

Theorem 2.14. If $G$ is a connected graph with $\operatorname{om}(G)=2$, then $f_{\text {om }}(G)=$ 0 .

Proof. Let $S=\{u, v\}$ be any minimum open monophonic set of $G$. Then by Theorem 1.2, $u$ and $v$ are the only extreme vertices such that every vertex of $G$ lies on a monophonic path joining $u$ and $v$. It follows from Theorem 1.1 that $S$ is the unique minimum open monophonic set of $G$. Hence by Theorem 2.5(i), $f_{\text {om }}(G)=0$.

Theorem 2.15. Given any positive integer with $n \geq 2$, there exists a connected graph $G$ of order $2 n$ such that $o m(G)=n$ and $f_{o m}(G)=0$.

Proof. Let $P_{n}$ be a path on $n$ vertices. Let $G$ be the graph obtained by adding a pendant edge at each vertex of $P_{n}$. Then it is clear that $o m(G)=n$ and $f_{\text {om }}(G)=0$.

The following graphs given in Figure 2.4 and Figure 2.5 are such that $o m(G)=2$. Hence by Theorem 2.14, $f_{\text {om }}(G)=0$ for these two graphs $G$.

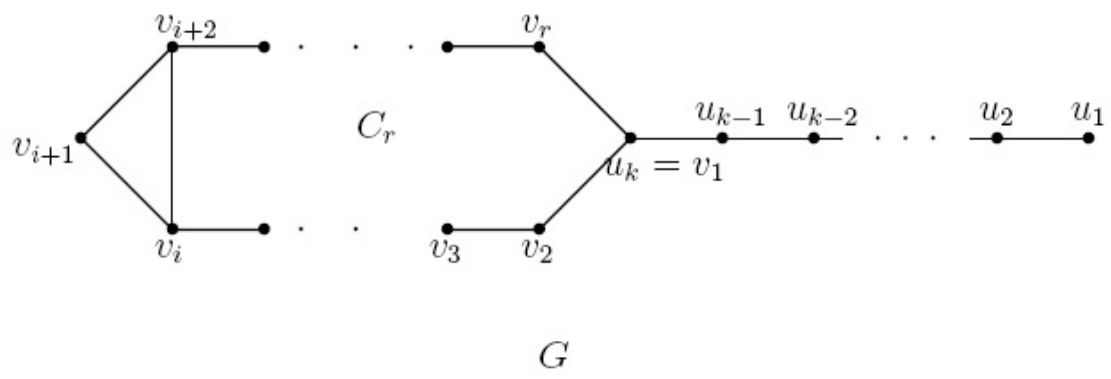

Figure 2.4 


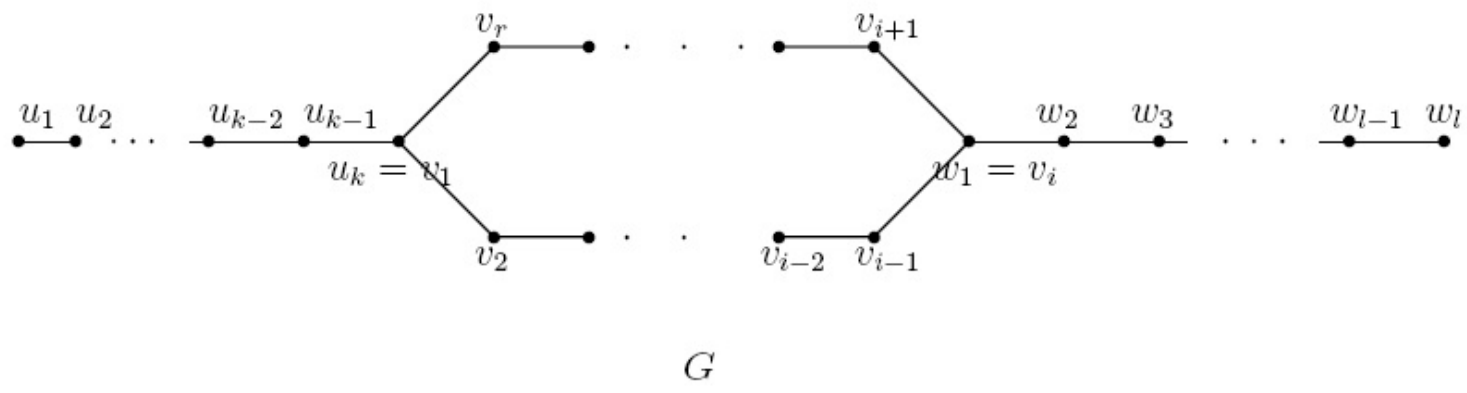

Figure 2.5.

In the view of Theorem 2.3, we have the following realization theorem.

Theorem 2.16. For every pair $a, b$ of integers with $0 \leq a \leq b-4$ and $b \geq 5$, there exists a connected graph $G$ such that $f_{o m}(G)=a$ and $o m(G)=b$.

Proof. We prove this theorem by considering three cases. For $b=4$, we have $a=0$. Hence for $G=K_{4}$, we have $f_{o m}(G)=0$ and $o m(G)=4=b$. Now, $b \geq 5$.

Case 1. $a=0$. Let $G=K_{b}$. Then, by Theorems 2.5(i) and 1.1, we have $f_{o m}(G)=0$ and $o m(G)=b$. 
Case 2. $a=1$.

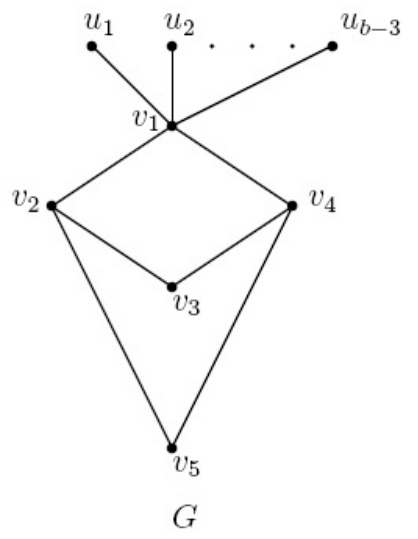

Figure 2.4.

Let $G$ be the graph in Figure 2.4, obtained from $C_{4}: v_{1}, v_{2}, v_{3}, v_{4}, v_{1}$ by first adding $b-3$ new vertices $u_{1}, u_{2}, u_{3}, \ldots, u_{b-3}$ to $C_{4}$ and joining each $u_{i}(1 \leq i \leq b-3)$ to the vertex $v_{1}$ of $C_{4}$; and also adding the new vertex $v_{5}$ and joining the edges $v_{5} v_{2}$ and $v_{5} v_{4}$. Let $S=\left\{u_{1}, u_{2}, \ldots, u_{b-3}\right\}$ be the set of all extreme vertices of $G$. By Theorem 1.1, every open monophonic set of $G$ contains $S$. Then $G$ contains exactly two minimum open monophonic sets namely $S_{1}=S \cup\left\{v_{2}, v_{3}, v_{4}\right\}$ and $S_{2}=S \cup\left\{v_{2}, v_{4}, v_{5}\right\}$. Thus om $(G)=|S|+$ $3=b$. Since $S_{1}$ is the unique minimum open monophonic set containing $v_{3}$, it follows from Theorem 2.5(ii) that $f_{\text {om }}(G)=1=a$.

Case 3. $a \geq 2$. Let $F_{i}: u_{i}, v_{i}, w_{i}$ be a path of order $3(1 \leq i \leq a)$. Let $P_{3}: u, v, w$ be a path of order 3 . Let $H$ be the graph obtained from the graphs $P_{3}$ and $F_{i}(1 \leq i \leq a)$ by first adding the $2 a$ edges $u u_{i}$ and $w w_{i}$ for $1 \leq i \leq a$; and also adding $b-a-2$ new vertices $y_{1}, y_{2}, \ldots, y_{b-a-3}, y_{b-a-2}$ and joining $y_{i}(1 \leq i \leq b-a-3)$ with $w$ and $y_{b-a-2}$ with $u$. Let $G$ be the graph in Figure 2.5 obtained from $H$ by adding the new vertex $x$ and joining each $u_{i}$ and $w_{i}(1 \leq i \leq a)$ with $x$, and also adding the new vertices $x_{1}$ and $x_{2}$ and joining the edges $x x_{1}$ and $v x_{2}$. We prove that $\operatorname{om}(G)=b$ and $f_{o m}(G)=a$. Let $S=\left\{x_{1}, x_{2}, y_{1}, y_{2}, \ldots, y_{b-a-3}, y_{b-a-2}\right\}$ be the set of all extreme vertices of $G$. By Theorem 1.1, every open monophonic set of $G$ contains $S$. Since the vertices $v_{1}, v_{2}, \ldots, v_{a}$ do not lie on a monophonic path joining any pair of vertices of $S, S$ is not an open monophonic set of $G$. Let $S_{i}=\left\{u_{i}, w_{i}\right\}(1 \leq i \leq a)$. We observe that every open monophonic set of $G$ must contain at least one vertex from each $X_{i}(1 \leq i \leq a)$. Thus 
$\operatorname{om}(G) \geq b-a+a=b$. Now, since the set $X=S \cup\left\{u_{1}, u_{2}, \ldots, u_{a}\right\}$ is an open monophonic set of $G$, it follows that $\operatorname{om}(G) \leq|X|=b$. Thus $\operatorname{om}(G)=b$. Next, we show that $f_{o m}(G)=a$. Since every open monophonic set of $G$ contains $S$, it follows from Theorem 2.7, that $f_{o m}(G) \leq o m(G)-|S|=$ $b-(b-a)=a$. Since $o m(G)=b$ and every open monophonic set of $G$ contains $S$, it is easily seen that every minimum open monophonic set $Y$ is of the form $S \cup\left\{z_{1}, z_{2}, \ldots, z_{n}\right\}$, where $z_{i} \in S_{i}(1 \leq i \leq a)$.

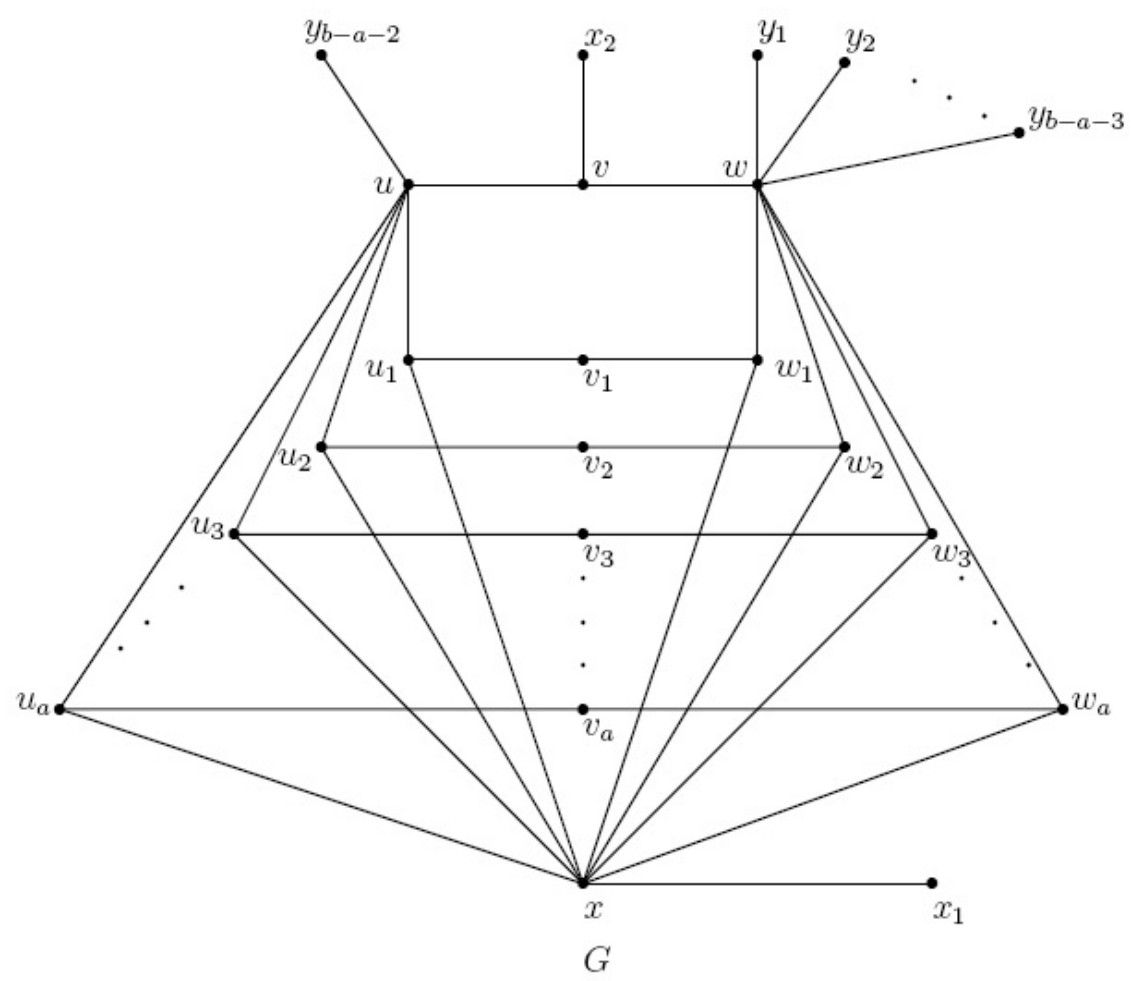

Figure 2.4.

Now, let $T$ be a proper subset of $Y$ with $|T|<a$. Then there is a vertex $z_{j}(1 \leq j \leq a)$ such that $z_{j} \notin T$. Let $t_{j}$ be a vertex of $S_{j}$ distinct from $z_{j}$. Then $Z=\left(Y-\left\{z_{j}\right\}\right) \cup\left\{t_{j}\right\}$ is an open monophonic set properly containing $T$. Thus $Y$ is not the unique minimum open monophonic set containing $T$. Thus $T$ is not a forcing subset of $Y$. This is true for all minimum open monophonic sets of $G$ and so it follows that $f_{o m}(G)=a$. 


\section{Addition of a pendant edge and forcing open monophonic number}

A main problem in graph theory that commonly arises is how the value of a parameter is affected by making a small change in the graph. In this section, we study how forcing open monophonic number of a graph is affected by the addition of a pendant edge. In this section we analyse how the forcing open monophonic numbers of certain standard graphs are affected by the addition of a pendant edge.

Theorem 3.1. For the graph $G=C_{n}+e(n \geq 3), o m(G)= \begin{cases}3 & \text { if } n \geq 5 \\ 4 & \text { if } n=4 \\ 3 & \text { if } n=3\end{cases}$ and $f_{\text {om }}(G)= \begin{cases}2 & \text { if } n \geq 6 \\ 0 & \text { if } n=3,4,5 .\end{cases}$

Proof. For the graph $G=C_{3}+e$ where $e=v_{1} x$, the set $S=\left\{x, v_{2}, v_{3}\right\}$ is the unique minimum open monophonic set of $G$ and so $\operatorname{om}(G)=3$, and by Theorem 2.5 (i), $f_{\text {om }}(G)=0$.

For the graph $G=C_{4}+e$, where $e=v_{1} x$, the set $S=\left\{x, v_{2}, v_{3}, v_{4}\right\}$ is the unique minimum open monophonic set of $G$ and so $\operatorname{om}(G)=4$, and by Theorem 2.5(i), $f_{\text {om }}(G)=0$.

For the graph $G=C_{5}+e$ where $e=v_{1} x$, the set $S=\left\{x, v_{3}, v_{4}\right\}$ is the unique minimum open monophonic set of $G$ and so $\operatorname{om}(G)=3$, and by Theorem 2.5(i), $f_{\text {om }}(G)=0$.

Let $C_{n}: v_{1}, v_{2}, v_{3}, \ldots, v_{n}, v_{1}$ be a cycle and let $G=C_{n}+e(n \geq 6)$ with $e=v_{i} x$. Let $v_{j}$ and $v_{k}$ be the vertices adjacent to $v_{i}$. Then it is clear that the $(n-3) C_{2}$ sets $S=\{x, u, v\}$ with $u$ and $v$ distinct from $v_{j}$ and $v_{k}$ are the only minimum open monophonic sets of $G$. It follows that $\operatorname{om}(G)=3$. Since no singleton subset of $S$ is a forcing subset for $S$ and since $\{u, v\}$ is the unique minimum forcing subset for $S$, it follows that $f_{o m}(G)=2$.

Theorem 3.2. Let $W_{n}=C_{n-1}+K_{1}(n \geq 5)$, where $C_{n-1}: v_{1}, v_{2}, \ldots, v_{n-1} v_{1}$ and $K_{1}=\left\{v_{n}\right\}$. Then for the graph $G=W_{n}+e$ where $W_{n}$ is a wheel on $n$ vertices and $e$ is the pendant edge added at a vertex on $C_{n-1}$,

$$
o m(G)=\left\{\begin{array}{cc}
3 \quad \text { if } n \geq 6 \\
4 & \text { if } n=5
\end{array} \text { and } f_{\text {om }}(G)=\left\{\begin{array}{cc}
2 & \text { if } n \geq 7 \\
0 & \text { if } n=5,6 .
\end{array}\right.\right.
$$


Proof. For the graph $G=W_{5}+e$, where $e=v_{1} x$, the set $S=$ $\left\{x, v_{2}, v_{3}, v_{4}\right\}$ is the unique minimum open monophonic set of $G$ so that $o m(G)=4$. Hence by Theorem 2.5(i), $f_{o m}(G)=0$.

For the graph $G=W_{6}+e$, where $e=v_{1} x$, the set $S=\left\{x, v_{3}, v_{4}\right\}$ is the unique minimum open monophonic set of $G$ so that $\operatorname{om}(G)=3$. Hence by Theorem 2.5(i), $f_{\text {om }}(G)=0$.

For the graph $G=W_{7}+e$, where $e=v_{1} x$, the sets $S_{1}=\left\{x, v_{3}, v_{4}\right\}$, $S_{2}=\left\{x, v_{3}, v_{5}\right\}$ and $S_{3}=\left\{x, v_{4}, v_{5}\right\}$ are the minimum open monophonic sets of $G$, it is follows that $o m(G)=3$. It is clear that the set $T_{1}=\left\{v_{3}, v_{4}\right\}$ is a minimum forcing subset of $S_{1}$ so that $f_{\text {om }}(G)=2$.

Let $G=C_{n}+e(n \geq 8)$ with $e=v_{i} x$. Let $v_{j}$ and $v_{k}$ be the vertices adjacent to $v_{i}$. Then it is clear that the $(n-3) C_{2}$ sets $S=\{x, u, v\}$ with $u$ and $v$ distinct from $v_{i}, v_{j}$ and $v_{k}$ are the only minimum open monophonic sets of $G$ so that $\operatorname{om}(G)=3$. Since no singleton subset of $S$ is a forcing subset for $S$ and since $\{u, v\}$ is the unique minimum forcing subset for $S$, it follows that $f_{\text {om }}(G)=2$.

Theorem 3.3. Let $W_{n}=C_{n-1}+K_{1}(n \geq 5)$, where $C_{n-1}: v_{1}, v_{2}, \ldots, v_{n-1} v_{1}$ and $K_{1}=\left\{v_{n}\right\}$. Then for the graph $G=W_{n}+e$ where $W_{n}$ is a wheel on $n$ vertices and $e$ is the pendant edge added at a vertex on $v_{n}$,

and

$$
\operatorname{om}(G)= \begin{cases}4 & \text { if } n \geq 7 \\ 5 & \text { if } n=5,6\end{cases}
$$

$$
f_{\text {om }}(G)= \begin{cases}3 & \text { if } n \geq 8 \\ 1 & \text { if } n=7 \\ 4 & \text { if } n=6 \\ 0 & \text { if } n=5\end{cases}
$$

Proof. Let $W_{n}=C_{n-1}+K_{1}$ where $C_{n-1}: v_{1}, v_{2}, \ldots, v_{n-1} v_{1}$ and $K_{1}=$ $\left\{v_{n}\right\}$.

For the graph $G=W_{5}+e$, where $e=v_{5} x$, the set $S=\left\{x, v_{1}, v_{2}, v_{3}, v_{4}\right\}$ is the unique minimum open monophonic set of $G$ so that $\operatorname{om}(G)=5$. By Theorem 2.5(i), $f_{\text {om }}(G)=0$.

For the graph $G=W_{6}+e$, where $e=v_{6} x$, the sets $S_{1}=\left\{x, v_{1}, v_{2}, v_{3}, v_{4}\right\}$, $S_{2}=\left\{x, v_{2}, v_{3}, v_{4}, v_{5}\right\}, S_{3}=\left\{x, v_{1}, v_{2}, v_{3}, v_{5}\right\}, S_{4}=\left\{x, v_{1}, v_{3}, v_{4}, v_{5}\right\}$ and $S_{5}=\left\{x, v_{1}, v_{2}, v_{4}, v_{5}\right\}$ are the unique minimum open monophonic sets of $G$ so that $\operatorname{om}(G)=5$. Since $T_{1}=\left\{v_{1}, v_{2}, v_{3}, v_{4}\right\}$ is a minimum forcing subset of $S_{1}$, it follows that $f_{o m}(G)=4$.

For the graph $G=W_{7}+e$, where $e=v_{7} x$, the sets $S_{1}=\left\{x, v_{1}, v_{3}, v_{5}\right\}$, $S_{2}=\left\{x, v_{2}, v_{4}, v_{6}\right\}$ are the only two minimum open monophonic sets of 
$G$, so that $\operatorname{om}(G)=4$. Since $\left\{v_{1}\right\}$ is a minimum forcing subset for $S_{1}$, it follows that $f_{\text {om }}(G)=1$.

Let $G=W_{n}+e(n \geq 8)$ with $e=v_{n} x$. Let $v_{i}, v_{j}, v_{k}$ be three nonadjacent vertices on $C_{n-1}$. Then it is clear that the sets $S=\left\{x, v_{i}, v_{j}, v_{k}\right\}$ are the only minimum open monophonic sets of $G$ so that $\operatorname{om}(G)=4$. Since $n \geq 8$, it is clear that no 2-element subset of any minimum open monophonic set $S$ is a forcing subset for $S$. Since $\left\{v_{i}, v_{j}, v_{k}\right\}$ is a forcing subset for $S$, it follows that $f_{\text {om }}(G)=3$.

Theorem 3.4. For the graph $G=K_{n}+e(n \geq 2)$, om $(G)=n$ and $f_{\text {om }}(G)=0$.

Proof. For the graph $G=K_{n}+e$, where $e=v_{1} x(n \geq 2)$, the set $S=$ $\left\{x, v_{2}, \ldots, v_{n}\right\}$ is the unique open monophonic set of $G$ so that $\operatorname{om}(G)=n$. By Theorem 2.5(i), $f_{\text {om }}(G)=0$.

We observe that for any non-trivial tree $T$, it is clear that $T+e$ is also a tree so that $f_{o m}(T+e)=0$.

Theorem 3.5. Let $U=\left\{u_{1}, u_{2}, \ldots, u_{r}\right\}$ and $W=\left\{w_{1}, w_{2}, \ldots, w_{s}\right\}$ be the partite sets of $K_{r, s}$. Then for the graph $G=K_{r, s}+e(2 \leq r \leq s)$, $o m(G)=4$,

and

$$
f_{\text {om }}(G)= \begin{cases}0 & \text { if } r=s=2 \\ 1 & \text { if } 3 \leq r \leq s\end{cases}
$$

for $2=r<s, f_{\text {om }}(G)=\left\{\begin{array}{lll}2 & \text { if } & e=u_{1} x \\ 1 & \text { if } & e=w_{1} x\end{array}\right.$

Proof. For $r=s=2$, we have $K_{r, s}=C_{4}$ and by Theorem 3.1, om $\left(K_{r, s}+\right.$ $e)=4$ and $f_{o m}\left(K_{r, s}+e\right)=0$.

For $2=r<s$, let $e=u_{1} x$. Then any set $S$ formed by taking $x, u_{2}$ along with any two elements $w_{i}, w_{j}$ of $W$ forms a minimum open monophonic set of $G$ so that $\operatorname{om}(G)=4$. It is clear that the subset $T=\left\{w_{i}, w_{j}\right\}$ is a forcing subset of $S$ and so $f_{o m}(G)=2$. Similarly, if $e=u_{2} x$, then again $\operatorname{om}(G)=4$ and $f_{o m}(G)=2$. Now, for $e=w_{1} x$, the sets $S_{i}=\left\{u_{1}, u_{2}, x, w_{i}\right\}\left(w_{i} \neq w_{1}\right)$ is the minimum open monophonic sets of $G$ so that $\operatorname{om}(G)=4$. Then $\left\{w_{i}\right\}$ is a forcing subset of $S_{i}$ so that $f_{o m}(G)=1$. Similarly, if $e=w_{i} x$, where $i \neq 1$, then again $\operatorname{om}(G)=4$ and $f_{\text {om }}(G)=1$.

For $3 \leq r \leq s$, let $e=u_{1} x$. Then a set $S_{i}$ formed by taking any two elements of $W$ together with $x$ and $u_{i}$ such that $i \neq 1$ forms a minimum 
open monophonic set. Hence $\operatorname{om}(G)=4$. Also $\left\{u_{i}\right\}$ is the forcing subset of $S_{i}$ so that $f_{o m}(G)=1$. Similarly, if $e=u_{i} x(i \neq 1)$ or $e=w_{i} x$, then again $o m(G)=4$ and $f_{o m}(G)=1$.

\section{Acknowledgements}

The authors are thankful to the referee for the useful suggestions for the revised version of this paper.

\section{References}

[1] F. Buckley and F. Harary, Distance in graphs, Addison-Wesley, Redwood city, CA, (1990).

[2] G. Chartrand, F. Harary, H. C. Swart and P. Zhang, Geodomination in graphs, Bulletin of the ICA, 31, pp. 51-59, (2001).

[3] G. Chartrand, E. M. Palmer and P. Zhang, The geodetic number of a graph: A survey, Congr. Numer., 156, pp. 37-58, (2002).

[4] W. Hale, Frequency Assignment; Theory and Applications, Proc. IEEE 68, pp. 1497-1514, (1980).

[5] F. Harary, Graph Theory, Addison-Wesley, (1969).

[6] R. Muntean and P. Zhang, On geodomination in graphs, Congr. Numer., 143, pp. 161-174, (2000).

[7] P. A. Ostrand, Graphs with specified radius and diameter, Discrete Math., 4, pp. 71-75, (1973).

[8] A. P. Santhakumaran and T. Kumari Latha, On the open geodetic number of a graph, SCIENTIA Series A: Mathematical Sciences, Vol. 20, pp. 131-142, (2010).

[9] A. P. Santhakumaran and M. Mahendran, The connected open monophonic number of a graph, International Journal of Computer Applications (0975-8887), Vol. 80 No. 1, pp. 39-42, (2013).

[10] A. P. Santhakumaran and M. Mahendran, The open monophonic number of a graph, International Journal of Scientific \& Engineering Research, Vol. 5 No. 2, pp. 1644-1649, (2014). 
[11] A. P. Santhakumaran and M. Mahendran, The total open monophonic number of a graph, Journal of Advances in Mathematics, Vol. 9 No. 3, pp. 2099-2107, (2014).

[12] A. P. Santhakumaran and M. Mahendran, The upper open monophonic number of a graph, Proyecciones Journal of Mathematics, Vol. 33 No. 4, pp. 389-403, (2014).

\author{
A. P. Santhakumaran \\ Department of Mathematics \\ Hindustan University \\ Hindustan Institute of Technology and Science \\ Chennai-603 103, \\ India \\ e-mail : apskumar1953@gmail.com \\ and \\ M. Mahendran \\ Department of Mathematics \\ Hindustan University \\ Hindustan Institute of Technology and Science \\ Chennai-603 103, \\ India \\ e-mail : magimani83@gmail.com
}

